\title{
SGB VIII-Reform und Inklusion
}

\author{
Wie inklusiv ist das neue Kinder- und Jugendstärkungsgesetz?
}

Das neue Kinder- und Jugendstärkungsgesetz ist mit dem Anspruch versehen, das SGB VIII inklusiver zu gestalten. Während einige Neuerungen bereits schon jetzt mit dessen Inkrafttreten enthalten sind, erfolgen wesentliche Änderungen hingegen erst ab 2028. Doch wie inklusiv sind diese Bemühungen tatsächlich?

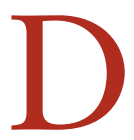
as SGB VIII wird seit seiner Entstehung im Jahr 1990 von der Debatte begleitet, die Zuständigkeit für Eingliederungshilfeleistungen für Kinder und Jugendliche mit Behinderung gänzlich in den Zuständigkeitsbereich der Kinder- und Jugendhilfe zu überführen. Bevor das SGB VIII auf den Weg gebracht wurde, war die gesamte Eingliederungshilfe für Menschen mit seelischer, geistiger oder körperlicher Behinderung ${ }^{1}$ Teil des Bundessozialhilfegesetzes ( $\mathbb{3} 39$ Abs. 1 BSHG).

Damals fiel die Entscheidung jedoch gegen diese sogenannte „Große Lösung“, da insbesondere Eltern von Kindern und Jugendlichen mit Behinderung sowie auch Behindertenverbände ihre Vorbehalte äußerten und Leistungseinbußen, eine Uminterpretation von behinderungsbedingten Bedarfen in Erziehungsbedarfe sowie eine Psychologisierung ihrer Anliegen befürchteten (Struck et al. 2010, S. 196). Zunächst als Teil der Hilfen zur Erziehung konzipiert (damals in $\$ 27$ Abs. 4 SGB VIII), wurde für die Eingliederungshilfen für seelisch behinderte Kinder und Jugendliche 1993 mit $\$ 35 a$ SGB VIII ein eigener Leistungstatbestand geschaffen, welcher bis heute rechtsgültig ist.

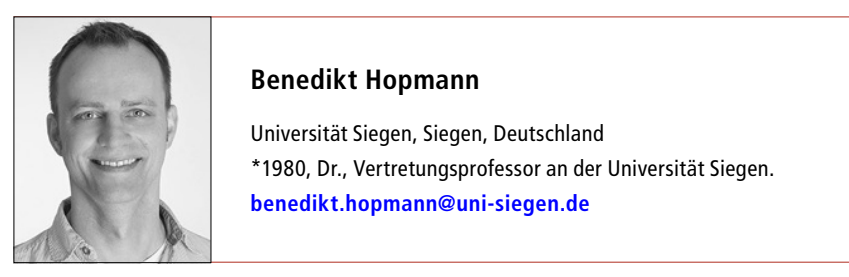

Zusammenfassung In diesem Beitrag werden wesentliche „,inklusive Schlaglichter“ des neuen Kinder- und Jugendstärkungsgesetzes hervorgehoben und diskutiert. Vor diesem Hintergrund werden schließlich zentrale Herausforderungen skizziert.

Schlüsselwörter Inklusion, SGB VIII-Reform, Kinder- und Jugendstärkungsgesetz
Demgegenüber war die Eingliederungshilfe bei körperlicher und geistiger Behinderung bis 31.12.2019 in den $\mathbb{S} \mathbb{S} 53 \mathrm{ff}$. SGB XII zu finden. Im Zuge der dritten Reformstufe des Bundesteilhabegesetzes ist sie seitdem in Teil 2 SGB IX kodifiziert. Diese als „Kleine Lösung " bezeichnete Konstruktion führt bis heute jedoch zu Schwierigkeiten: So sind erziehungsbedingte und behinderungsbedingte Bedarfe - insbesondere in der frühen Kindheit - nicht immer eindeutig voneinander abzugrenzen und Mehrfachbehinderungen gestatten nur schwerlich eine klare Aufspaltung in geistige und seelische Behinderung. Diese Unklarheiten führen bis heute zu Zuständigkeitsstreitigkeiten zwischen Jugend- und Sozialhilfeträgern und somit zur Verzögerung oder sogar Verwehrung von Leistungen (Meysen 2014, S. 221). Durch den 13. Kinder- und Jugendbericht (BMFSFJ 2009) und die durch Deutschland im Jahr 2009 ratifizierte UN-Behindertenrechtskonvention (UN-BRK) hat die Debatte wieder an Fahrt aufgenommen. Neuordnungsvorschläge wurden schließlich durch die BundLänder-AG „Inklusion von jungen Menschen mit Behinderung " erarbeitet (ASMK und JFMK 2013). Mit dem ab 2017 in der ersten Reformstufe in Kraft getretenen Bundesteilhabegesetz (BTHG) geht der Anspruch einher, den Implikationen der UN-BRK gerecht zu werden (Rohrmann 2019). Unabhängig davon, ob dieser Anspruch tatsächlich eingelöst werden konnte, hat das BTHG bereits unabhängig von einer Umsetzung der Gesamtzuständigkeit Konsequenzen für die Kinder- und Jugendhilfe. Bedeutsam mit Blick auf die Kinder- und Jugendhilfe ist die Einführung des Teilhabeplanverfahrens nach $\mathbb{1} 19$ SGB IX, welches erst noch in ein Verhältnis zur Hilfeplanung nach $\mathbb{3} 36$ SGB VIII zu setzen ist, die Frage nach Instrumenten der Bedarfsermittlung/klärung sowie der seit 01.01.2018 - inspiriert durch die UN-BRK - um die „Wechselwirkung mit einstellungs- und umweltbedingten Barrieren “ ergänzte Behinderungsbegriff ( $\$ 2$ Abs. 1 SGB IX). 
Im Koalitionsvertrag der 18. Legislaturperiode wurde schließlich ein Reformvorhaben angekündigt, „das jahrelange politische und fachliche sowie höchst strittige Auseinandersetzungen in der Kinder- und Jugendhilfe bündeln sollte“ (Böllert 2017, S. 9). Zwischen 2015 und 2016 kursierten erst inoffizielle und später auch offizielle Gesetzesentwürfe, die jedoch am 09.11.2016 durch das BMFSFJ zurückgezogen wurden. Dem gingen zahlreiche kritische Einwürfe ob der Intransparenz und der inhaltlichen Ausrichtung voraus (z. B. Ziegler 2016). Nachdem das am 29.06.2017 vom Bundestag beschlossene Kinder- und Jugendstärkungsgesetz (KJSG) im Bundesrat scheiterte und es ohnehin keine nennenswerten Bemühungen um Inklusion mehr enthielt, findet sich im Koalitionsvertrag der 19. Legislaturperiode die Absichtserklärung, „das Kinder- und Jugendhilferecht auf Basis des in der letzten Legislaturperiode beschlossenen Kinder- und Jugendstärkungsgesetzes weiter[zu] entwickeln" (CDU et al. 2018, S. 21). Das mittlerweile unter der Programmatik der „Inklusiven Lösung“ diskutierte Reformvorhaben wurde von 11/2018 bis 12/2019 als Dialogprozess Mitreden - Mitgestalten: Die Zukunft der Kinder-und Jugendhilfe weitergeführt (BMFSFJ 2020). Am Ende des Gesetzgebungsprozesses ist das neue Kinder- und Jugendstärkungsgesetz am 07.05.2021verabschiedet worden und am 10.06.2021 (im Wesentlichen) in Kraft getreten.

Mit dem neuen Kinder- und Jugendstärkungsgesetz gelangt der langwierige und strittige SGB VIII-Reformprozess nunmehr zu einem vorläufigen Ende. Vor diesem Hintergrund wird im Folgenden diskutiert, welche Inklusionsperspektive das neue SGB VIII bereits jetzt schon mit Inkrafttreten bietet und welche hingegen bis 2028 erfolgen sollen. Diese Fokussierung auf den Inklusionsgehalt des neuen Gesetzes soll jedoch nicht darüber hinwegtäuschen, dass daneben noch weitere Schwerpunktthemen enthalten sind, die mindestens ebenso intensiv zu diskutieren sind (siehe z. B. DGfE-Kommission Sozialpädagogik 2020 für eine inhaltliche Stellungnahme zum gesamten Referentenentwurf sowie die anderen Beiträge in diesem Heft). Im Folgenden werden das Gehalt des Inklusions- und Teilhabeverständnisses, Fragen der (De-)Kategorisierung, die Herausforderung einer inklusiven Hilfeplanung sowie das Potenzial der zukünftigen „Inklusiven Lösung“ ab 2028 genauer in den Blick genommen.

\section{Teilhabe von Individuen oder Individualisierung von Teilhabe?}

Die Teilhabe- und Inklusionspostulate im neuen SGB VIII sind als ambivalent zu beurteilen. Grundsätzlich ist es zu würdigen und zudem längst überfällig, dass diese vor dem Hintergrund der allgemeinen Entwicklungspfade und Diskurse von Inklusion (dazu ausführlicher Hopmann 2021) im neuen SGB VIII erstmalig Berücksichtigung finden. Gleichwohl ist fraglich, welche Reichweite damit erlangt wird. Denn „so wichtig die Ziele der gleichberechtigten Teilhabe und des Abbaus von Barrieren sind, so sicher ist, dass pädagogische Fachkräfte weder den Kindern eine gleichberechtigte Teilhabe anerziehen, noch Barrieren aberziehen können " (Zinsmeister 2021, S. 138). Im neuen Gesetz aber kommt ein solches interaktionistisches und individualistisches Verständnis von Teilhabe zur Anwendung.

Während Inklusion begrifflich kaum im neuen Gesetz vorkommt (lediglich als Adjektiv in $\mathbb{7} 77$ Abs. $1, \mathbb{S} 79 \mathrm{a}$ und $\mathbb{S} 80$ Abs. 2), geht es hier maßgeblich um Teilhabe. Ein Blick in das neue Gesetz offenbart deutlich das Teilhabeverständnis, welches dem Vorhaben zugrunde liegt. „Jugendhilfe soll zur Verwirklichung des Rechts nach Absatz 1 insbesondere [...] jungen Menschen ermöglichen oder erleichtern, entsprechend ihrem Alter und ihrer individuellen Fähigkeiten in allen sie betreffenden Lebensbereichen selbstbestimmt zu interagieren und damit gleichberechtigt am Leben in der Gesellschaft teilhaben zu können“ ( $\$ 1$ Abs. 3 Nr. 2 SGB VIII). Darin spiegelt sich das interaktionistische und individualistische Verständnis von Teilhabe ${ }^{2}$ wider, welches in der Begründung nochmals bekräftigt wird. „Teilhabe wird dabei als Möglichkeit zu einer dem Alter und den individuellen Fähigkeiten entsprechenden selbstbestimmten Interaktion in allen junge Menschen betreffenden Lebensbereichen verstanden " (Deutscher Bundestag 2021, S. 67 Begründung zu Art. 1 Nr. 2b).

Überraschend an dieser Stelle ist, dass dieses Verständnis von Teilhabe selbst hinter den Anspruch der Kinder- und Jugendhilfe zurückfällt (unabhängig davon, wie weitreichend dieser zu bewerten ist) „dazu bei[zu] tragen, positive Lebensbedingungen für junge Menschen und ihre Familien sowie eine kinder- und familienfreundliche Umwelt zu erhalten oder zu schaffen" ( $\ 1$ Abs. 3 Nr. 5 SGB VIII). Mit dieser Ausrichtung verfehlt das Teilhabeverständnis nicht zuletzt auch das gesellschaftskritische Ansinnen, wofür die Integrationsund Inklusionsbewegungen jahrzehntelang kämpf $(\mathrm{t}) \mathrm{en}$ (Feuser 2012).

In einem ähnlich ambivalenten Zusammenhang ist auch der Selbstbestimmungsbegriff zu bewerten, welcher erstmalig in $\mathbb{} 1$ Abs. 1 SGB VIII Einzug erhalten hat. Denn so positiv dieses Selbstbestimmungsversprechen auch zu bewerten ist und so sehr es auch mit den Forderungen von Selbsthilfebewegungen wie insbesondere der Selbstbestimmt-Leben-Bewegung korrespondiert (Weber 2010), so kommt es nun darauf 
an, inwiefern sich Kindern und Jugendlichen dadurch tatsächlich mehr Selbstbestimmungspotential eröffnet (Schröer 2021) oder ob sich dieses Selbstbestimmungsversprechen nicht eher als individualisierte „neoliberale Pflicht“ (Waldschmidt 2012, S. 32) entpuppt, selbstbestimmt leben zu müssen (Rohrmann 2018, S. 625).

\section{(De-)Kategorisierungsarbeit?}

Im Kinder- und Jugendstärkungsgesetz sind die Umgangsweisen mit Bedarfskategorien im Kontext der erzieherischen Hilfen und der Eingliederungshilfen von hoher Relevanz. Trotz der inhaltlich unterschiedlichen Ausrichtung von erzieherischem Bedarf einerseits und behinderungsbedingtem Unterstützungsbedarf andererseits ist beiden grundsätzlich gemein, dass diesen „subjektive Mangeldefinitionen “ (Halfar 2017, S. 80) vorausgehen, welche überwiegend mit einer stigmatisierenden Bedürftigkeitsprüfung (Schrödter 2020) einhergehen.

Die Grundausrichtung des KJSG hat dabei eine Überarbeitung erfahren. Nunmehr heißt es folgendermaßen: „Bei der Ausgestaltung der Leistungen und der Erfüllung der Aufgaben sind [...] die unterschiedlichen Lebenslagen von Mädchen, Jungen sowie transidenten, nichtbinären und intergeschlechtlichen jungen Menschen zu berücksichtigen, Benachteiligungen abzubauen und die Gleichberechtigung der Geschlechter zu fördern, die gleichberechtigte Teilhabe von jungen Menschen mit und ohne Behinderungen umzusetzen und vorhandene Barrieren abzubauen “ ( $\$ 9$ Nr. 3 \& 4 SGB VIII).

Bemerkenswert ist an dieser Stelle, dass im neuen SGB VIII die Dichotomie von Mädchen/Jungen aufgehoben wurde und nun verschiedene Geschlechtsidentitäten Berücksichtigung finden sollen. Warum hinsichtlich der Teilhabe nun allerdings wieder auf die Dichotomie Behinderung/Nicht-Behinderung verwiesen wird, welche zudem von den vorherigen Forderungen der Gleichberechtigung bzw. Aufhebung von Benachteiligung abgekoppelt wird, ist inhaltlich auf den ersten Blick nicht nachvollziehbar. Nachvollziehbar wird es jedoch auf den zweiten Blick bei einer Betrachtung der dahinterstehenden Konstruktionslogik, welche auf den sozialrechtlichen Behinderungsbegriff ( $\mathbb{S} 2$ Abs. 1 SGB IX $)^{3}$ verweist. Trotz der bereits erwähnten Erweiterung um die Wechselwirkungen mit einstellungs- und umweltbedingten Barrieren überwindet dieses Behinderungsverständnis Kausalitätsannahmen zwischen Beeinträchtigung und Behinderung nach wie vor nicht (Rohrmann 2018, S. 627). Diese Überwindung aber wäre sowohl für einen weitreichenden Inklusions- und Teilhabebegriff als auch für ein umfassenderes Verständnis von Behinderung äußerst entscheidend. Denn nicht nur fällt dieser Begriff von Behinderung damit hinter längst geführte Diskurse zurück (Dederich 2009), sondern es bleibt vor allem Teilhabe zuförderst mit der Behinderungskategorie verknüpft. Schließlich ist demnach eine Einschränkung der Teilhabe obne zugrunde liegende Beeinträchtigung diesem Modell nach nicht denkbar. Die Formulierung der „spezifischen Bedürfnisse“ ( $\sqrt{ } 77$ Abs. 1 SGB VIII) betont zudem die Abweichung von Kindern und Jugendlichen mit Behinderung von der Norm nichtbehinderter Kinder und Jugendlichen und fördert somit eine „ableistische Dominanzkultur“ (Zinsmeister 2021, S. 140) im Sinne einer Individualisierung ungleicher Verhältnisse.

Durch das BTHG sind der Behinderungsbegriff und das damit verknüpfte Teilhabeverständnis vorerst gesetzt, so dass der Möglichkeit und Ermöglichung von Inklusion (kategoriale) Grenzen gesetzt werden. Daher vermag auch die geplante Zusammenführung der Systeme nicht die Notwendigkeit der kategorialen Zuordnung zu überwinden (Molnar et al. 2021).

\section{Inklusive Hilfeplanung: Partizipative Bedarfs-} klärung oder objektivistische Bedarfsermittlung?

Die Intensivierung der Fachdebatte über die Konzeptentwicklung zur partizipativen Verständigung über individuelle Bedarfe und Hilfen ist zukünftig als Kernherausforderung einer inklusiven Kinder- und Jugendhilfe zu verstehen (Hopmann et al. 2020). Damit einher geht einerseits die Verhältnisbestimmung der Hilfeplanung nach $\mathbb{3} 36$ SGB VIII sowie der Teilhabeplanung nach $\$ 19$ SGB IX und andererseits die Frage nach dem konkreten Verfahren. Mit Blick auf Ersteres sind Vor- und Nachrangigkeit der jeweiligen Verfahren derzeit noch in der Diskussion (Schönecker 2019, S. 44). Letzteres wird im KJSG dadurch aufgenommen, dass „sicherzustellen [ist], dass Beratung und Aufklärung nach Satz 1 in einer für den Personensorgeberechtigten und das Kind oder den Jugendlichen verständlichen, nachvollziehbaren und wahrnehmbaren Form erfolgen “ ( $\$ 36$ Abs. 1 SGB VIII). Obgleich an dieser Stelle verfahrensbezogene Offenheit suggeriert wird, welche im Vergleich zu den restriktiv-bürokratischen Vorschlägen des vorherigen gescheiterten Reformanlaufs sicherlich eine eindeutige Errungenschaft darstellt (Ziegler 2016), ist kaum zu verkennen, dass die $\mathrm{ICF}^{4}$ mittlerweile als Assessmentinstrument überhöht wird (Hopmann et al. 2020, S. 343), welches dem Anspruch einer beteiligungsorientierten ${ }^{5}$, angesichts deutungsbedürftiger Situationen auf prozessorientiertes Fallverstehen ausgerichteten Hilfeplanungspraxis zuwiderläuft. Durch das BTHG sind die Vorgaben zur Bedarfsermittlung nach $\mathbb{} 13$ SGB IX jedenfalls bereits für die Eingliederungshilfen nach $\$ 35$ a SGB VIII verbindlich. Es ist nun für die Ausgestaltung einer inklu- 
siven Kinder- und Jugendhilfe entscheidend, eine inklusive Hilfeplanung konzeptionell voranzubringen. Die parallelen Prozesse von BTHG und SGB VIII Reform haben bislang jedenfalls eher zu einer Auseinanderentwicklung geführt (Rohrmann 2021, S. 58).

\section{Warten auf die "Inklusive Lösung"?}

Die „Inklusive Lösung “ selbst ist im KJSG bislang nur als zukünftige Absichtserklärung enthalten. Die $\mathrm{Zu}$ sammenführung der Rechtskreise und Leistungen für Kinder und Jugendliche mit und ohne Behinderung soll im Jahre 2028 erfolgen, jedoch in Abhängigkeit eines vorab zu verabschiedenden Bundesgesetzes bis 2027 (\$ 107 SGB VIII). Bis dahin sollen Verfahrenslots_innen bei Eingliederungshilfeleistungen ab 2024 zum Einsatz kommen, um Eltern sowie Kinder und Jugendliche bei allen Verfahrensschritten zu begleiten $(\mathbb{S} 10 \mathrm{~b}$ SGB VIII). Diese Perspektive sendet einerseits ein positives Signal, die Zuständigkeitsstreitigkeiten zwischen den Leistungsträgern bald überwunden zu haben. Andererseits geht damit aber auch eine Innovationsbremse einher aufgrund der gesetzlichen Festschreibung, „den leistungsberechtigten Personenkreis, Art und Umfang der Leistungen sowie den Umfang der Kostenbeteiligung für die hierzu Verpflichteten nach dem am 1. Januar 2023 für die Eingliederungshilfe geltenden Recht beizubehalten“ ( $\$ 107$ Abs. 2 SGB VIII). Vor diesem Hintergrund bleibt nun auszuloten, ob und wenn ja, welcher Spielraum nun bis 2028 besteht und wie dieser bereits jetzt gestaltet werden kann. Zukunftsweisende Einzelprojekte wie das Projekt „Inklusion jetzt!“ (Hollweg und Kieslinger 2020) oder das Zukunftsforum Heimerziehung (Schönecker et al. 2021) haben hier bereits wichtige Vorarbeit geleistet. Trotz der in diesem Beitrag aufgezeigten Limitierungen sind die durchaus bestehenden Gestaltungsspielräume daher schon jetzt zu nutzen und fachliche Konzepte und Instrumente weiterzuentwickeln. Denn die Ausgestaltung des Bundesgesetzes bis 2027 wird entscheidend davon abhängig sein, ob bzw. welche Dynamik sich bis dahin entfaltet.

„Die „Inklusion“ hat die Kinder- und Jugendhilfe längst erreicht - doch alle warten auf die „große Lösung “" - so lautet der Titel eines Beitrags von Oehme und Schröer aus dem Jahr 2014 (Oehme und Schröer 2014). Es wäre fatal, nun auf die „Inklusive Lösung““ im Jahr 2028 zu warten.

Eingegangen. 9. August 2021

Angenommen. 15. September 2021
Funding. Open Access funding enabled and organized by Projekt DEAL.

Open Access. Dieser Artikel wird unter der Creative Commons Namensnennung 4.0 International Lizenz veröffentlicht, welche die Nutzung, Vervielfältigung, Bearbeitung, Verbreitung und Wiedergabe in jeglichem Medium und Format erlaubt, sofern Sie den/die ursprünglichen Autor(en) und die Quelle ordnungsgemäß nennen, einen Link zur Creative Commons Lizenz beifügen und angeben, ob Änderungen vorgenommen wurden.

Die in diesem Artikel enthaltenen Bilder und sonstiges Drittmaterial unterliegen ebenfalls der genannten Creative Commons Lizenz, sofern sich aus der Abbildungslegende nichts anderes ergibt. Sofern das betreffende Material nicht unter der genannten Creative Commons Lizenz steht und die betreffende Handlung nicht nach gesetzlichen Vorschriften erlaubt ist, ist für die oben aufgeführten Weiterverwendungen des Materials die Einwilligung des jeweiligen Rechteinhabers einzuholen.

Weitere Details zur Lizenz entnehmen Sie bitte der Lizenzinformation auf http://creativecommons.org/licenses/by/4.0/deed.de.

1. Damit wird ausschließlich auf das sozialrechtliche Behinderungsverständnis rekurriert, welches nicht zwangsläufig deckungsgleich ist mit sozial-und kulturwissenschaftlichen Fachdebatten um die Kategorie der Behinderung (Dederich 2009).

2. Damit korrespondiert auch die Rede von „Fähigkeiten zur aktiven Teilhabe "in $\$ 16$ zur allgemeinen Förderung der Erziehung in der Familie sowie „Entwicklungs-, Teilhabe- oder Erziehungsbedingungen in der Herkunftsfamilie" in Abs. 1 \$ 37 zur Beratung und Unterstützung von Eltern.

3. Während die Begriffsbestimmung von Behinderung in $\int 7$ Abs. 2 $S G B$ VIII im Sinne der Begrifflichkeit im SGB IX angepasst wurde, ist diese in $\int 35 a$ Abs. 1 SGB VIII jedoch nicht erfolgt.

4. Die International Classification of Functioning, Disability and Health (ICF) ist eine Klassifikation der Weltgesundheitsorganisation (WHO), die Behinderung als biopsychosoziales Modell versteht und vor diesem Hintergrund verschiedene Teilhabedimensionen beschreibt. Sie ist jedoch kein Diagnose- oder Bedarfsklärungsinstrument (vgl. Hopmann et al. 2020, S. 343).

5. Obgleich Anspruch und Wirklichkeit immer noch auseinandergeben (Pluto 2018, S. 955).

\section{Literatur}

ASMK, \& JFMK (2013). Bericht der von der ASMK und JFMK eingesetzten Arbeitsgruppe „Inklusion von jungen Menschen mit Behinderung “. www.bag-if.de/wp-content/uploads/2013/04/Abschlussbericht_Endfassung. pdf. Zugegriffen: 17. Aug. 2021.

BMFSFJ (2009). 13. Kinder- und Jugendbericht. Bericht über die Lebenssituation junger Menschen und die Leistungen der Kinder- und Jugendhilfe in Deutschland. http://dip21.bundestag.de/dip21/btd/16/128/1612860.pdf. Zugegriffen: 17. Aug. 2021.

BMFSFJ (2020). Abschlussbericht. Mitreden - Mitgestalten: Die Zukunft der Kinder- und Jugendhilfe. https://www.mitreden-mitgestalten.de/sites/default/files/downloads/abschlussbericht-mitreden-mitgestalten-die-zukunftder-kinder-und-jugendhilfe-data.pdf. Zugegriffen: 17. Aug. 2021.

Böllert, K. (2017). SGB VIII-Reform - Eine never ending story mit ungewissem Ausgang. Widersprüche, 37(4), 9-18.

CDU, CSU, \& SPD (2018). Ein neuer Aufbruch für Europa. Eine neue Dynamik für Deutschland. Ein neuer Zusammenhalt für unser Land. Koalitionsvertrag zwischen CDU, CSU und SPD. Koalitionsvertrag der 19. Legislaturperiode vom 12. März 2018. https://www.bundesregierung. de/resource/blob/974430/847984/5b8bc23590d4cb2892b31c987ad67 2b7/2018-03-14-koalitionsvertrag-data.pdf. Zugegriffen: 17. Aug. 2021. 
Dederich, M. (2009). Behinderung als sozial- und kulturwissenschaftliche Kategorie. In M. Dederich \& W. Jantzen (Hrsg.), Behinderung und Anerkennung. Behinderung, Bildung, Partizipation - Enzyklopädisches Handbuch der Behindertenpädagogik (Bd. 2, S. 15-39). Stuttgart: Kohlhammer.

Deutscher Bundestag (2021). Drucksache 19/26107. 19. Wahlperiode, 25.01.2021. Gesetzentwurf der Bundesregierung. Entwurf eines Gesetzes zur Stärkung von Kindern und Jugendlichen (Kinder- und Jugendstärkungsgesetz - KJSG). http://dip21.bundestag.de/dip21/btd/19/261/1926107.pdf. Zugegriffen: 17. Aug. 2021.

DGfE-Kommission Sozialpädagogik (2020). Stellungnahme des Vorstandes der Kommission Sozialpädagogik in der DGfE zum Referentenentwurf eines Gesetzes zur Stärkung von Kindern und Jugendlichen (KJSG-RefE 2020). https://www.dgfe.de/fileadmin/OrdnerRedakteure/Sektionen/Sek08_ SozPaed/KSozPaed/2020_Stellungnahme_Sozialpaedagogik_RefEntwurf_ KJSG.pdf. Zugegriffen: 17. Aug. 2021.

Feuser, G. (2012). Der lange Marsch durch die Institutionen. Ein Inklusionismus war nicht das Ziel! Behindertenpädagogik, 51(1), 5-34.

Halfar, B. (2017). Bedarf. In Deutscher Verein für öffentliche und private Fürsorge e. V. (Hrsg.), Fachlexikon der Sozialen Arbeit (8. Aufl. S. 79-80). Baden-Baden: Nomos.

Hollweg, C., \& Kieslinger, D. (2020). Das Modellprojekt Inklusion jetzt! Inklusionsorientierte Erziehungshilfe: Perspektiven, Herausforderungen, Lösungsansätze. frühe Kindheit, 04, 70-73.

Hopmann, B. (2021). Vergewisserungen zum Inklusionsbegriff. In D. Kieslinger \& C. Hollweg (Hrsg.), Hilfeplanung inklusiv gedacht. Ansätze, Perspektiven, Konzepte (S. 23-44). Freiburg i. Br.: Lambertus.

Hopmann, B., Rohrmann, A., Schröer, W., \& Urban-Stahl, U. (2020). SGB VIII-Reform: Quo vadis Hilfe- und Teilhabeplanung mit jungen Menschen und ihren Eltern? Das Jugendamt - Zeitschrift für Jugendhilfe und Familienrecht, 93(7/8), 338-346.

Meysen, T. (2014). Gesamtzuständigkeit im SGB VIII. neue praxis, 44(3), 220-232.

Molnar, D., Oehme, A., Renker, A., \& Rohrmann, A. (2021). Kategorisierungsarbeit in Hilfen für Kinder und Jugendliche mit und obne Behinderung. Eine vergleichende Untersuchung. Weinheim/Basel: Beltz Juventa.

Oehme, A., \& Schröer, W. (2014). Die „Inklusion“ hat die Kinder- und Jugendhilfe längst erreicht - doch alle warten auf die „große Lösung“. In Arbeitsgemeinschaft für Kinder- und Jugendhilfe (AGJ) (Hrsg.), Gesellschaftlicher Wandel - Neue Herausforderungen für die Kinder- und Jugendhilfe?! (S. 25-38). Berlin: AGJ.

Pluto, L. (2018). Partizipation und Beteiligungsrechte. In K. Böllert (Hrsg.), Kompendium Kinder- und Jugendhilfe (S. 945-965). Wiesbaden: Springer VS.

Rohrmann, A. (2019). Das Bundesteilhabegesetz - Ausdruck eines Paradigmenwechsels? Archiv für Wissenschaft und Praxis der sozialen Arbeit, 50(1), 4-14.

Rohrmann, A. (2021). Die Entwicklung der Hilfeplanung in der Kinderund Jugendhilfe und in der Unterstützung von Menschen mit Behinderungen. In D. Kieslinger \& C. Hollweg (Hrsg.), Hilfeplanung inklusiv gedacht. Ansätze, Perspektiven, Konzepte (S. 45-65). Freiburg i. Br.: Lambertus.

Rohrmann, E. (2018). Zwischen selbstbestimmter sozialer Teilhabe, fürsorglicher Ausgrenzung und Bevormundung. Ausgewählte Lebenslagen von Menschen, die wir behindert nennen. In E.-U. Huster, J. Boeckh \& H. Mogge-Grotjahn (Hrsg.), Handbuch Armut und soziale Ausgrenzung (3. Aufl. S. 619-640). Wiesbaden: VS

Schönecker, L. (2019). Rechtsfragen bei der Instrumentenentwicklung aufgrund der Änderungen durch das Bundesteilhabegesetz (BTHG). In Teilhabebeeinträchtigungen bei Kindern und Jugendlichen mit (drohender) seelischer Behinderung erkennen (S. 24-53). Ulm: Klinik für Kinder- und Jugendpsychiatrie/Psychotherapie des Universitätsklinikums Ulm. Rechtliche Anforderungen an Einschätzungen nach Änderungen durch das Bundesteilhabegesetz und Vorstellung eines darauf abgestimmten Instruments für die Jugendhilfe.

Schönecker, L., Seckinger, M., Eisenhardt, B., Kuhn, A., van Driesten, A., Hahne, C., Horn, J., Strüder, H., \& Koch, J. (2021). Inklusive Weiterentwicklung außerfamiliärer Wohnformen für junge Menschen mit Behinderungen im Rahmen der Initiative „Zukunftsforum Heimerziehung“. Diskussionspapier. https://igfh.de/sites/default/files/2021-02/UAG_Inklusion_Heimerziehung_Web.pdf. Zugegriffen: 17. Aug. 2021.

Schrödter, M. (2020). Bedingungslose Jugendhilfe. Von der selektiven Abhilfe defizitärer Elternschaft zur universalen Unterstützung von Erziehung. Wiesbaden: Springer VS.
Schröer, W. (2021). „Stärkere Selbstbestimmung durch das KJSG“ - Werden die jungen Menschen den Unterschied merken? Das Jugendamt - Zeitschrift für Jugendhilfe und Familienrecht, 94(7/8), 354-358.

Struck, N., Porr, C., \& Koch, J. (2010). Zeit lassen... aber ganz schnell anfangen! Zur aktuellen Debatte um die „Große Lösung“. Forum Erziehungshilfen, 16(4), 196-201.

Waldschmidt, A. (2012). Selbstbestimmung als Konstruktion. Alltagstheorien behinderter Frauen und Männer (2. Aufl.). Wiesbaden: VS.

Weber, E. (2010). Selbstbestimmung. In Enzyklopädie Erziehungswissenschaft Online (S. 1-24).

Ziegler, H. (2016). Sozialpädagogik vs. SGB VIII Reform. neue praxis, 46(5), 491-499.

Zinsmeister, J. (2021). Inklusion und assistierte Autonomie: Zum Rechtsstatus von Kindern und Jugendlichen in der UN-Behindertenrechtskonvention. In K. Scheiwe, W. Schröer, F. Wapler \& M. Wrase (Hrsg.), Der Rechtsstatus junger Menschen im Kinder- und Jugendhilferecht. Beiträge zum ersten Forum Kinder- und Jugendhilferecht (S. 123-161). Baden-Baden: Nomos. 